7 August 2000

\title{
Resonance production in central $p p$ collisions at the CERN Omega Spectrometer
}

\author{
A. Kirk \\ School of Physics and Astronomy, University of Birmingham, Birmingham, U.K.
}

\begin{abstract}
A study of resonance production in central $p p$ collisions is presented as a function of several kinematical variables. In particular the difference in the transverse momentum $\left(d P_{T}\right)$ of the exchanged particles shows that undisputed $q \bar{q}$ mesons are suppressed at small $d P_{T}$ whereas glueball candidates are enhanced and in addition, the azimuthal angle $\phi$ gives information on the nature of the Pomeron.
\end{abstract}

Submitted to Physics Letters 
The Omega central production experiments (WA76, WA91 and WA102) were designed to study exclusive final states formed in the reaction

$$
\mathrm{pp} \longrightarrow \mathrm{p}_{f} X^{0} \mathrm{p}_{s}
$$

where the subscripts $f$ and $s$ refer to the fastest and slowest particles in the laboratory frame respectively and $X^{0}$ represents the central system. Such reactions are expected to be mediated by double exchange processes where both Pomeron and Reggeon exchange can occur. The trigger was designed to enhance double exchange processes with respect to single exchange and elastic processes. Details of the trigger conditions, the data processing and event selection have been given in previous publications [1].

The experiments have been performed at incident beam momenta of 85,300 and $450 \mathrm{GeV} / \mathrm{c}$, corresponding to centre-of-mass energies of $\sqrt{s}=12.7,23.8$ and $29 \mathrm{GeV}$. Theoretical predictions [2] of the evolution of the different exchange mechanisms with centre of mass energy, $\sqrt{s}$, suggest that

$$
\begin{gathered}
\sigma(\mathrm{RR}) \sim s^{-1} \\
\sigma(\mathrm{R} \mathbb{P}) \sim s^{-0.5} \\
\sigma(\mathbb{P} \mathbb{P}) \sim \text { constant }
\end{gathered}
$$

where RR, R $\mathbb{P}$ and $\mathbb{P} \mathbb{P}$ refer to Reggeon-Reggeon, Reggeon-Pomeron and Pomeron-Pomeron exchange respectively. Hence we expect Double Pomeron Exchange (DPE) to be more significant at high energies, whereas the Reggeon-Reggeon and Reggeon-Pomeron mechanisms will be of decreasing importance. The decrease of the non-DPE cross section with energy can be inferred from data taken by the WA76 collaboration using pp interactions at $\sqrt{s}$ of $12.7 \mathrm{GeV}$ and $23.8 \mathrm{GeV}$ [3]. The $\pi^{+} \pi^{-}$mass spectra for the two cases show that the signal-to-background ratio for the $\rho^{0}(770)$ is much lower at high energy, and the WA76 collaboration report that the ratio of the $\rho^{0}(770)$ cross sections at $23.8 \mathrm{GeV}$ and $12.7 \mathrm{GeV}$ is $0.44 \pm 0.07$. Since isospin 1 states such as the $\rho^{0}(770)$ cannot be produced by DPE, the decrease of the $\rho^{0}(770)$ signal at high $\sqrt{s}$ is consistent with DPE becoming relatively more important with increasing energy with respect to other exchange processes.

Due to the fact that there was no electromagnetic calorimeter in the WA76 experiment at $\sqrt{s}$ of $12.7 \mathrm{GeV}$, the $s$ dependence of only a small number of states can be determined. The states for which the $s$ dependence have been measured and whose compatibility with DPE have been determined are given in table 1. Although there was no calorimeter in the $85 \mathrm{GeV} / \mathrm{c}$ run of the WA76 experiment, it was able to reconstruct the $\eta \pi^{+} \pi^{-}$mass spectrum using the decay $\eta \rightarrow \pi^{+} \pi^{-}\left(\pi^{0}\right)_{\text {missing }}$ [4]. In this mass spectrum the $\eta^{\prime}$ and $f_{1}(1285)$ are seen. The cross section of the $f_{1}(1285)$ at $85 \mathrm{GeV} / \mathrm{c}$ has been well measured through its all charged particle decay modes and hence can be used to determine the cross section of the $\eta^{\prime}$.

After correcting for geometrical acceptances, detector efficiencies, losses due to selection cuts, and all known decay modes, the cross-sections for the production of all the resonances at $\sqrt{s}=29.1 \mathrm{GeV}$ observed in the WA102 experiment are shown in table 1. As can be seen, there is evidence that in central $p p$ collisions $s \bar{s}$ production is much weaker than $n \bar{n}$ production. For example, the cross section for the production of the $f_{2}(1270)$, whose production has been found to be consistent with DPE, is more than 40 times greater than the cross section of the $f_{2}^{\prime}(1525)$. Part of this suppression could be due to the fact that there is a kinematic suppression of $M_{X^{0}}^{-2}$ 
and some could be explained by the fact that the centre of mass energy dependence of the $f_{2}(1270)$ allows for up to a $30 \%$ contribution from non-DPE processes. However, a suppression of $\approx 20$ still needs to be explained. Hence there could be some strong dependence on the mass of the produced quarks in DPE. A similar effect is observed in electroproduction $(\gamma \mathbb{P})$ where the $\phi$ becomes more suppressed relative to the $\rho$ at low $\mathrm{Q}^{2}$ of the photon [5].

The cross section as a function of mass has not previously been shown for the centrally produced system. This distribution could be determined in two ways. Either by measuring the missing mass from the two outgoing protons or by measuring the effective mass of the decay products of the central system. The latter method has the disadvantage of requiring a separate acceptance correction for each final state. However, due to the measurement errors on the outgoing fast proton, the second method has the overwhelming advantage of having more than an order of magnitude better resolution in the determination of the mass. In order to determine the $d \sigma / d M_{X_{0}}$ differential cross section, starting with the $\pi \pi$ final state, the acceptance corrected mass spectra of all the final states observed in the WA102 experiment have been added together and the resulting differential cross section, $d \sigma / d M_{X_{0}}$, is shown in fig. 1 1 a). Prominent features are the $\eta, \omega, \eta^{\prime}, f_{1}(1285), f_{1}(1420)$ and a broad enhancement around $2 \mathrm{GeV}$.

In addition to the cross section and $s$ dependences, there are a number of different kinematical variables that can be determined for each resonance. The first is the Feynman $x\left(x_{F}\right)$ distribution of the central system. It should be noted that for resonances that decay into all charged particle decay modes the $x_{F}$ distribution measured is symmetric about 0 . However, for resonances that decay into final states including $\gamma \mathrm{s}$, because of the acceptance of the calorimeter, only events with $x_{F}>0$. are detected. Hence for consistency, only $x_{F}>0$ will be shown. All the resonances observed tend to fall into two distinct classes: fig. 2a) those that are peaked at $x_{F}=0$ and fig. $2 \mathrm{~b}$ ) those that are peaked away from 0 . All the resonances that can be produced by DPE (i.e. those with $\mathrm{I}=0$ and $\mathrm{G}$ parity positive) fall into the first class while those with $\mathrm{I}=1$ and/or $\mathrm{G}$ parity negative fall into the second class which may be due to the fact that, in part, they are produced by diffraction from the proton vertex rather than from being due to real central events. Fig. 1 b) shows the $d \sigma / d M_{X_{0}}$, distribution for $\left|x_{F}\right|<0.1$. As can be seen the $\omega$, which can not be produced by DPE and which has a $x_{F}$ distribution peaked away from zero, is suppressed in the $\left|x_{F}\right|<0.1$ distribution relative to the total sample.

Another variable that can be measured is the four momentum transfer squared $(|t|)$ at one of the proton vertices. The $|t|$ distribution has been measured for all the resonances. All the distributions fall into three distinct classes as summarised in fig. 2c)-e). Fig. 2c) (type I) has the classic shape associated with $\pi$ exchange [4] and has been fitted by

$$
\frac{d \sigma}{d t}=\alpha \exp b_{1} t+\beta t \exp b_{2} t
$$

where $\alpha$ and $\beta$ are found to be similar in size. Fig. 2d) (type II) is similar to spin flip distributions and can be fitted by

$$
\frac{d \sigma}{d t}=\alpha t \exp b_{2} t
$$

Fig. 2e) (type III) represents non-spin flip distributions and can be fitted by

$$
\frac{d \sigma}{d t}=\alpha \exp b_{1} t
$$


The results of the fit to $d \sigma / d t$ and the $t$ distributions types for all the resonances observed are given in table 11.

In addition to the kinematical variables discussed above, in central production there are variables that are specific to the central vertex at which the two exchanged particles interact to form the final state $X^{0}$. The WA91 collaboration published a paper [6] showing that there was a difference in the resonances observed when the angle between the outgoing slow and fast protons was near to 0 degrees compared to when the angle was near to 180 degrees. In order to try to explain this effect in terms of a physical model, Close and Kirk [7] proposed that the data be analysed in terms of the parameter $d P_{T}$, which is the difference in transverse momentum between the particles exchanged from the fast and slow vertices.

The WA102 collaboration has presented studies of how different resonances are produced as a function of the parameter $d P_{T}$ [8]. The fraction of each resonance has been calculated for $d P_{T} \leq 0.2 \mathrm{GeV}, 0.2 \leq d P_{T} \leq 0.5 \mathrm{GeV}$ and $d P_{T} \geq 0.5 \mathrm{GeV}$. In addition, the ratio of production at small $d P_{T}$ to large $d P_{T}$ has been calculated. The results are presented in table 2 for all the resonances observed in the WA102 experiment. Figure 3 shows the ratio of the number of events for $d P_{T}<0.2 \mathrm{GeV}$ to the number of events for $d P_{T}>0.5 \mathrm{GeV}$ for each resonance considered. It can be observed that all the undisputed $q \bar{q}$ states which can be produced in DPE, namely those with positive $G$ parity and $I=0$, have a very small value for this ratio $(\leq 0.1)$. Some of the states with $I=1$ or $\mathrm{G}$ parity negative, which can not be produced by DPE, have a slightly higher value $(\approx 0.25)$. However, all of these states are suppressed relative to the interesting states, which could have a gluonic component, which have a large value for this ratio. Fig. 1c) shows the $d \sigma / d M_{X_{0}}$ distribution for $d P_{T}<0.2$ and fig. 11d) the $d \sigma / d M_{X_{0}}$ distribution for $d P_{T}>0.5$. The dominant feature of the distribution for $d P_{T}<0.2$ is the low mass peak which comes from the $\pi \pi$ system. This $\pi \pi$ mass spectrum is dominated by the $\mathrm{S}$-Wave although there is a $10 \% \mathrm{D}$-wave contribution [9]. The $\mathrm{S}$ and $\mathrm{D}$-waves both have the same $d P_{T}$ distributions which are similar to those observed for the $f_{0}(980)$.

In addition to the $d P_{T}$ dependencies, an interesting effect has been observed in the azimuthal angle $\phi$ which is defined as the angle between the $p_{T}$ vectors of the two outgoing protons. Historically it has been assumed that the Pomeron, with "vacuum quantum numbers", transforms as a scalar and hence that the $\phi$ distribution would be flat for resonances produced by DPE. The $\phi$ dependences observed [8] are clearly not flat and considerable variation is observed among the resonances produced. The $\phi$ distributions for all the resonances observed in the WA102 experiment are shown in figs. 4, 5. These azimuthal dependences, as a function of $J^{P C}$, are very striking. For the mesons that can be produced by DPE the $\phi$ distributions maximise around $90^{\circ}$ for resonances with $J^{P C}=0^{-+}$, at $180^{\circ}$ for those with $J^{P C}=1^{++}$and at $0^{\circ}$ for those with $J^{P C}=2^{-+}$. As can be seen from fig. 5 the $\phi$ distributions are not simply a J-dependent effect, since $0^{++}$production peaks at $0^{\circ}$ for some states whereas others are more evenly spread; moreover, $2^{++}$established $q \bar{q}$ states peak at $180^{\circ}$ whereas the $f_{2}(1910)$ and $f_{2}(1950)$ peak at $0^{\circ}$.

Several theoretical papers have been published on these effects [10, 11]. All agree that the exchanged particle must have $\mathrm{J}>0$ and that $\mathrm{J}=1$ is the simplest explanation for the observed $\phi$ distributions. Close and Schuler [11] have calculated the $\phi$ dependences for the production of resonances with different $J^{P C}$ for the case where the exchanged particle is a Pomeron that transforms like a non-conserved vector current. In fact this is the only model in which detailed 
calculations are made for resonances with spins other than $J^{P C}=0^{-+}$. In order to gain insight into the nature of the particles exchanged in central $p p$ interactions Close, Kirk and Schuler [12] have compared the predictions of this model with the data for resonances with different $J^{P C}$ observed in the WA102 experiment. They found that for the production of $J^{P C}=0^{-+}$mesons they could predict the $\phi$ dependence and the vanishing cross section as $t \rightarrow 0$ absolutely and fit the $t$ slope in terms of one parameter, $b_{T}$. For the $J^{P C}=1^{++}$mesons they could predict the general form for the $\phi$ distribution. By fitting the $t$ slope they obtained the parameter $b_{L}$; this then gave a parameter free prediction for the variation of the $\phi$ distribution as a function of $t$ which agrees with the data. In addition, they obtained absolute predictions for the $t$ and $\phi$ dependences of the $J^{P C}=2^{-+}$mesons which were again in accord with the data when helicity 1 dominance was imposed. In the $0^{++}$and $2^{++}$sector they were able to fit all the $\phi$ distributions with one parameter $\left(\mu^{2}\right)$ and they found that it is primarily the sign of this quantity that drives the $\phi$ dependences. Understanding the dynamical origin of this sign is now a central issue in the quest to distinguish $q \bar{q}$ states from glueballs or other exotic states.

In a recent paper by Close and Kirk [13] studying the mixing of the scalar glueball with the nearby $q \bar{q}$ states it was noted that the $\phi$ dependencies of the $f_{0}(1370), f_{0}(1500)$ and $f_{0}(1710)$, and hence the sign of $\left(\mu^{2}\right)$, could be explained if they were due to the relative phase between the $n \bar{n}$ and $g g$ in their wavefunctions.

One interesting feature is that there is very little evidence for radially excited states in central $p p$ collisions. Therefore it is even more striking that there are three tensor states in the region of $2 \mathrm{GeV}$ which is where the tensor glueball is predicted to be by Lattice QCD [14]. Two of these states have similar $\phi$ dependencies and one the opposite. This effect is very similar to what was observed in the scalar sector. It is interesting to speculate whether the $f_{2}(1910)$, $f_{2}(1950)$ and $f_{2}(2150)$ are due to mixing between a tensor glueball and nearby $q \bar{q}$ states. This may explain their observation in central production as being through their gluonic component.

In summary, a study of the production of resonances in central $p p$ interactions has been presented. Cuts on the $d P_{T}$ variable select out known $q \bar{q}$ states from glueball candidates. Why this works is still to be understood. The azimuthal angle $\phi$ has given information on the nature of the Pomeron. This information may in turn be used in future to extract information on the gluonic content of the observed mesons. It is interesting to note that although there is strong evidence for all the ground state nonet states (except for those with $J^{P C}=1^{+-}$) there is little evidence for radially excited nonet members. This may give special significance to the observation of the $f_{2}(1910), f_{2}(1950)$ and $f_{2}(2150)$.

\section{Acknowledgements}

This work is supported, in part, by grants from the British Particle Physics and Astronomy Research Council and the British Royal Society. The author wishes to thank the WA76, WA91 and WA102 collaborations for the use of their data. 


\section{References}

[1] T.A. Armstrong et al., Nucl. Instr. and Methods A274 (1989) 165;

F. Antinori et al., Il Nuovo Cimento A107 (1994) 1857.

[2] S.N. Ganguli and D.P. Roy, Phys. Rep. 67 (1980) 203.

[3] T.A.Armstrong et al., Zeit. Phys. C 51 (1991) 351.

[4] D. Barberis et al., Phys. Lett. B467 (1999) 165.

[5] C. Adloff et al. (H1 Collaboration), Phys. Lett. B483 (2000) 360.

[6] D. Barberis et al., Phys. Lett. B388 (1996) 853.

[7] F.E. Close and A. Kirk, Phys. Lett. B397 (1997) 333.

[8] D. Barberis et al., Phys. Lett. B422 (1998) 399;

D. Barberis et al., Phys. Lett. B427 (1998) 398;

D. Barberis et al., Phys. Lett. B432 (1998) 436;

D. Barberis et al., Phys. Lett. B436 (1998) 204;

D. Barberis et al., Phys. Lett. B440 (1998) 225;

D. Barberis et al., Phys. Lett. B462 (1999) 462;

D. Barberis et al., Phys. Lett. B471 (2000) 435;

D. Barberis et al., Phys. Lett. B474 (2000) 423;

D. Barberis et al., Phys. Lett. B479 (2000) 59.

[9] D. Barberis et al., Phys. Lett. B453 (1999) 316.

[10] F.E. Close, Phys. Lett. B419 (1998) 387;

P. Castoldi, R. Escribano and J.-M. Frere, Phys. Lett. B425 (1998) 359;

N. I. Kochelev, hep-ph/9902203;

N.I. Kochelev, T. Morii and A.V. Vinnikov, Phys. Lett. B457 (1999) 202;

F.E. Close and G. Schuler, Phys. Lett. B458 (1999) 127.

[11] F.E. Close and G. Schuler, Phys. Lett. B464 (1999) 279.

[12] F.E. Close, A. Kirk and G. Schuler, Phys. Lett. B477 (2000) 13.

[13] F.E. Close and A. Kirk, Phys. Lett. B483 (2000) 345.

[14] G. Bali et al. (UKQCD), Phys. Lett. B309 (1993) 378;

D. Weingarten, Nucl. Phys. Proc. Suppl. 53 (1997) 232;

J. Sexton et al., Phys. Rev. Lett. 75 (1995) 4563;

F.E. Close and M.J. Teper, "On the lightest Scalar Glueball" Rutherford Appleton Laboratory report no. RAL-96-040;

W. Lee and D. Weingarten, hep-lat/9805029;

G. Bali, hep-ph/0001312 
Table 1: Summary of resonance production. $t$ type I, II and III refer to the shape of the $|t|$ distribution as depicted in fig. 2 2c), d) and e) respectively. The error quoted represents the statistical and systematic errors summed in quadrature.

\begin{tabular}{|c|c|c|c|c|c|c|}
\hline$J^{P C}$ & Resonance & $\begin{array}{c}\sigma(\mathrm{nb}) \\
\text { at } \sqrt{s}=29.1 \\
\mathrm{GeV}\end{array}$ & $t$ type & $\begin{array}{l}\text { Value of } b_{1} \text { in } \\
\qquad \exp -b_{1} t \\
\left(\mathrm{GeV}^{-2}\right)\end{array}$ & $\begin{array}{l}\text { Value of } b_{2} \text { in } \\
\qquad t \exp -b_{2} t \\
\quad\left(\mathrm{GeV}^{-2}\right)\end{array}$ & $\begin{array}{c}\text { DPE } \\
\text { Compatible }\end{array}$ \\
\hline $0^{-+}$ & $\begin{array}{c}\pi^{0} \\
\eta \\
\eta^{\prime}\end{array}$ & $\begin{array}{c}22011 \pm 3267 \\
3859 \pm 368 \\
1717 \pm 184\end{array}$ & $\begin{array}{l}\text { I } \\
\text { II } \\
\text { II }\end{array}$ & $17.3 \pm 0.2$ & $\begin{array}{c}17.6 \pm 0.3 \\
8.2 \pm 0.3 \\
8.1 \pm 0.4\end{array}$ & Yes \\
\hline $0^{++}$ & $\begin{array}{c}a_{0}(980) \\
f_{0}(980) \\
f_{0}(1370) \\
f_{0}(1500) \\
f_{0}(1710) \\
f_{0}(2000)\end{array}$ & $\begin{array}{c}638 \pm 60 \\
5711 \pm 450 \\
1753 \pm 580 \\
2914 \pm 301 \\
245 \pm 65 \\
3139 \pm 480\end{array}$ & $\begin{array}{l}\text { III } \\
\text { III } \\
\text { III } \\
\text { III } \\
\text { III } \\
\text { III }\end{array}$ & $\begin{array}{l}6.2 \pm 0.8 \\
5.3 \pm 0.2 \\
6.7 \pm 0.2 \\
5.2 \pm 0.5 \\
6.5 \pm 0.5 \\
5.6 \pm 0.4\end{array}$ & & $\begin{array}{l}\text { Yes } \\
\text { Yes }\end{array}$ \\
\hline $1^{++}$ & $\begin{array}{l}a_{1}(1260) \\
f_{1}(1285) \\
f_{1}(1420)\end{array}$ & $\begin{array}{c}10011 \pm 900 \\
6857 \pm 1306 \\
1080 \pm 385\end{array}$ & $\begin{array}{l}\text { III } \\
\text { III } \\
\text { III }\end{array}$ & $\begin{array}{l}6.9 \pm 0.2 \\
6.3 \pm 0.3 \\
5.6 \pm 0.5\end{array}$ & & $\begin{array}{l}\text { Yes } \\
\text { Yes }\end{array}$ \\
\hline $1^{--}$ & $\begin{array}{l}\rho(770) \\
\omega(782) \\
\phi(1020)\end{array}$ & $\begin{array}{c}3102 \pm 250 \\
7440 \pm 553 \\
60 \pm 21\end{array}$ & $\begin{array}{l}\text { III } \\
\text { I } \\
\text { III }\end{array}$ & $\begin{array}{l}5.8 \pm 0.1 \\
3.8 \pm 0.2 \\
7.8 \pm 1.0\end{array}$ & $24.5 \pm 0.6$ & $\begin{array}{l}\text { No } \\
\text { No }\end{array}$ \\
\hline $2^{-+}$ & $\begin{array}{l}\pi_{2}(1670) \\
\eta_{2}(1645) \\
\eta_{2}(1870)\end{array}$ & $\begin{array}{l}1505 \pm 145 \\
1907 \pm 152 \\
1940 \pm 185\end{array}$ & $\begin{array}{l}\text { I } \\
\text { II } \\
\text { II }\end{array}$ & $4.3 \pm 0.2$ & $\begin{array}{c}22.3 \pm 1.0 \\
7.3 \pm 1.3 \\
8.3 \pm 2.0\end{array}$ & \\
\hline $2^{++}$ & $\begin{array}{l}a_{2}(1320) \\
f_{2}(1270) \\
f_{2}^{\prime}(1520) \\
f_{2}(1910) \\
f_{2}(1950) \\
f_{2}(2150)\end{array}$ & 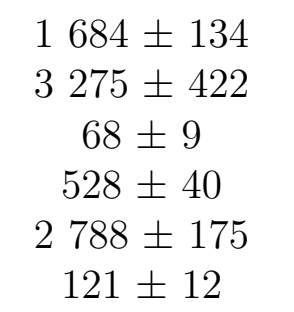 & $\begin{array}{l}\text { III } \\
\text { II } \\
\text { II } \\
\text { III } \\
\text { III } \\
\text { III }\end{array}$ & $\begin{array}{l}7.8 \pm 0.3 \\
4.9 \pm 1.6 \\
5.9 \pm 0.4 \\
5.4 \pm 1.4\end{array}$ & $\begin{array}{l}8.7 \pm 0.4 \\
7.7 \pm 3.3\end{array}$ & Yes \\
\hline
\end{tabular}


Table 2: Resonance production as a function of $d P_{T}$ expressed as a percentage of its total contribution. The error quoted represents the statistical and systematic errors summed in quadrature.

\begin{tabular}{|c|c|c|c|c|c|}
\hline$J^{P C}$ & Resonance & $d P_{T} \leq 0.2 \mathrm{GeV}$ & $0.2 \leq d P_{T} \leq 0.5 \mathrm{GeV}$ & $d P_{T} \geq 0.5 \mathrm{GeV}$ & $\mathrm{R}$ \\
\hline $0^{-+}$ & $\begin{array}{c}\pi^{0} \\
\eta \\
\eta^{\prime}\end{array}$ & $\begin{array}{c}12 \pm 2 \\
6 \pm 2 \\
3 \pm 2\end{array}$ & $\begin{array}{l}45 \pm 2 \\
34 \pm 2 \\
32 \pm 2\end{array}$ & $\begin{array}{l}43 \pm 2 \\
60 \pm 3 \\
64 \pm 3\end{array}$ & $\begin{array}{l}0.27 \pm 0.05 \\
0.10 \pm 0.03 \\
0.05 \pm 0.03\end{array}$ \\
\hline $0^{++}$ & $\begin{array}{c}a_{0}(980) \\
f_{0}(980) \\
f_{0}(1370) \\
f_{0}(1500) \\
f_{0}(1710) \\
f_{0}(2000)\end{array}$ & $\begin{array}{l}25 \pm 4 \\
23 \pm 2 \\
18 \pm 4 \\
24 \pm 2 \\
26 \pm 2 \\
12 \pm 2\end{array}$ & $\begin{array}{c}33 \pm 5 \\
51 \pm 3 \\
32 \pm 2 \\
54 \pm 3 \\
46 \pm 2 \\
38 \pm 3\end{array}$ & $\begin{array}{l}42 \pm 6 \\
26 \pm 3 \\
50 \pm 3 \\
22 \pm 4 \\
28 \pm 2 \\
50 \pm 4\end{array}$ & $\begin{array}{l}0.57 \pm 0.14 \\
0.88 \pm 0.12 \\
0.36 \pm 0.08 \\
1.05 \pm 0.18 \\
0.95 \pm 0.10 \\
0.23 \pm 0.04\end{array}$ \\
\hline $1^{++}$ & $\begin{array}{l}a_{1}(1260) \\
f_{1}(1285) \\
f_{1}(1420)\end{array}$ & $\begin{array}{l}13 \pm 3 \\
3 \pm 1 \\
2 \pm 2\end{array}$ & $\begin{array}{l}51 \pm 4 \\
35 \pm 2 \\
38 \pm 2\end{array}$ & $\begin{array}{l}36 \pm 3 \\
61 \pm 4 \\
60 \pm 4\end{array}$ & $\begin{array}{l}0.36 \pm 0.09 \\
0.05 \pm 0.02 \\
0.03 \pm 0.03\end{array}$ \\
\hline $1^{--}$ & $\begin{array}{c}\rho(770) \\
\omega(782) \\
\phi(1020)\end{array}$ & $\begin{array}{c}6 \pm 2 \\
10 \pm 2 \\
8 \pm 3\end{array}$ & $\begin{array}{l}41 \pm 4 \\
40 \pm 2 \\
47 \pm 3\end{array}$ & $\begin{array}{l}53 \pm 3 \\
49 \pm 3 \\
45 \pm 4\end{array}$ & $\begin{array}{l}0.12 \pm 0.05 \\
0.20 \pm 0.04 \\
0.18 \pm 0.07\end{array}$ \\
\hline $2^{-+}$ & $\begin{array}{l}\pi_{2}(1670) \\
\eta_{2}(1645) \\
\eta_{2}(1870)\end{array}$ & $\begin{array}{l}11 \pm 2 \\
9 \pm 1 \\
8 \pm 1\end{array}$ & $\begin{array}{l}48 \pm 4 \\
32 \pm 3 \\
29 \pm 3\end{array}$ & $\begin{array}{l}40 \pm 4 \\
59 \pm 5 \\
63 \pm 6\end{array}$ & $\begin{array}{c}0.27 \pm 0.06 \\
0.15 \pm 0.02 \\
0.13 \pm 0.02\end{array}$ \\
\hline $2^{++}$ & $\begin{array}{l}a_{2}(1320) \\
f_{2}(1270) \\
f_{2}^{\prime}(1520) \\
f_{2}(1910) \\
f_{2}(1950) \\
f_{2}(2150)\end{array}$ & $\begin{array}{c}10 \pm 2 \\
8 \pm 1 \\
4 \pm 3 \\
20 \pm 4 \\
27 \pm 2 \\
3 \pm 3\end{array}$ & $\begin{array}{l}38 \pm 5 \\
29 \pm 1 \\
36 \pm 3 \\
62 \pm 7 \\
46 \pm 5 \\
53 \pm 4\end{array}$ & $\begin{array}{l}52 \pm 6 \\
63 \pm 2 \\
60 \pm 4 \\
18 \pm 4 \\
27 \pm 2 \\
44 \pm 3\end{array}$ & $\begin{array}{c}0.19 \pm 0.05 \\
0.12 \pm 0.02 \\
0.07 \pm 0.04 \\
1.1 \pm 0.3 \\
1.0 \pm 0.12 \\
0.06 \pm 0.08\end{array}$ \\
\hline
\end{tabular}




\section{Figures}

Figure 1: The differential cross section, $d \sigma / d M_{X_{0}}$, of the centrally produced system $X^{0}$ for a) all data, b) $\left|x_{F}\right|<0.1$, c) $d P_{T}<0.2 \mathrm{GeV}$ and d) $d P_{T}>0.5 \mathrm{GeV}$.

Figure 2: The $\left|x_{F}\right|$ distribution for a) the $f_{2}(1270)$ and $\mathrm{b}$ ) the $\omega$. The $|t|$ distributions for $\left.\mathrm{c}\right)$ the $\left.\pi^{0}, \mathrm{~d}\right)$ the $\eta^{\prime}$ and e) the $f_{1}(1285)$.

Figure 3: The ratio of the number of events with $d P_{T} \leq 0.2$ to the number of events with $d P_{T} \geq 0.5 \mathrm{GeV}$ for all the resonances observed grouped in $J^{P C}$.

Figure 4: The azimuthal angle $\phi$ between the fast and slow protons for resonances with $J^{P C}=0^{-+}, 1^{--}, 1^{++}$and $2^{-+}$.

Figure 5: The azimuthal angle $\phi$ between the fast and slow protons for resonances with $J^{P C}=0^{++}$and $2^{++}$. 

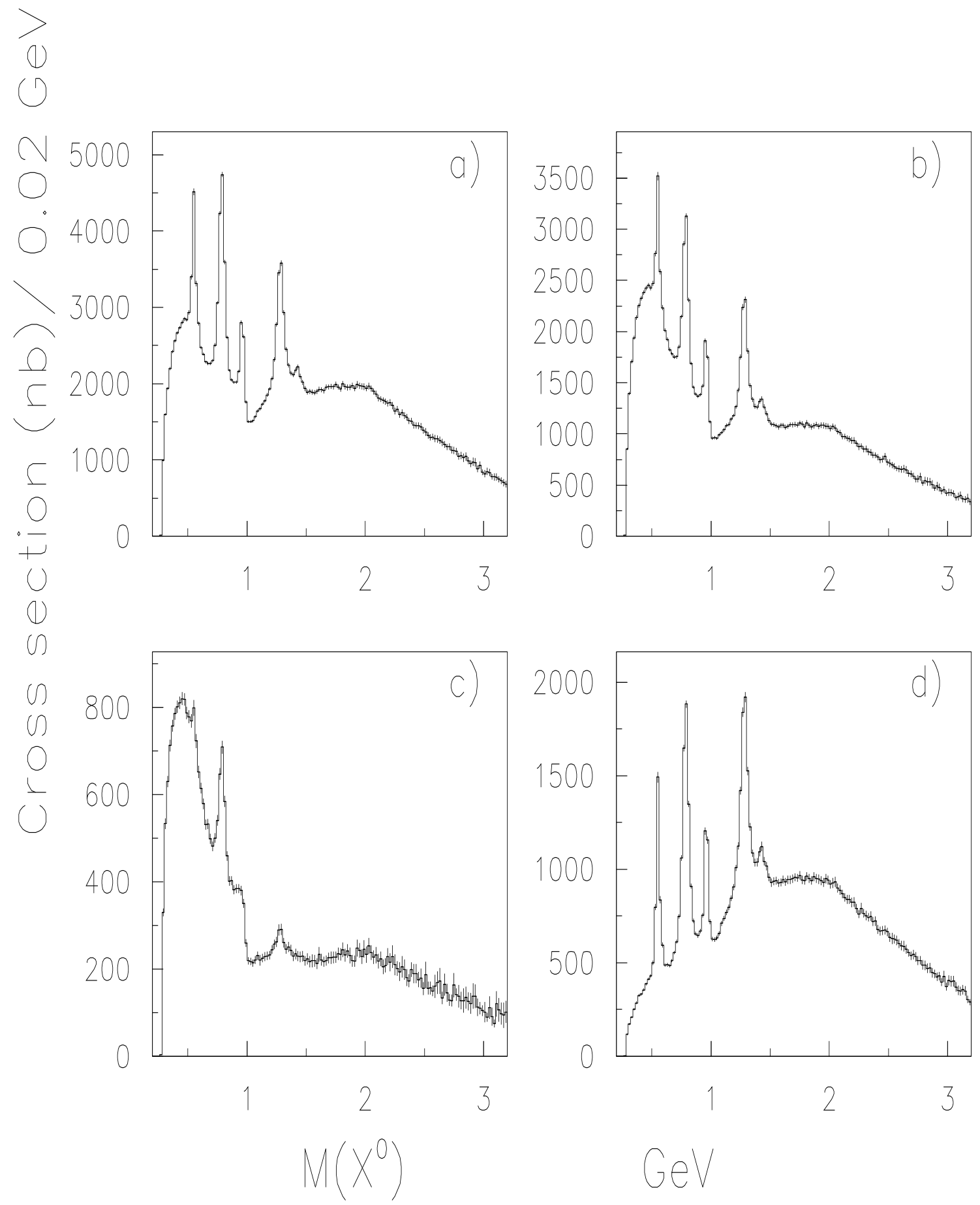

Figure 1 

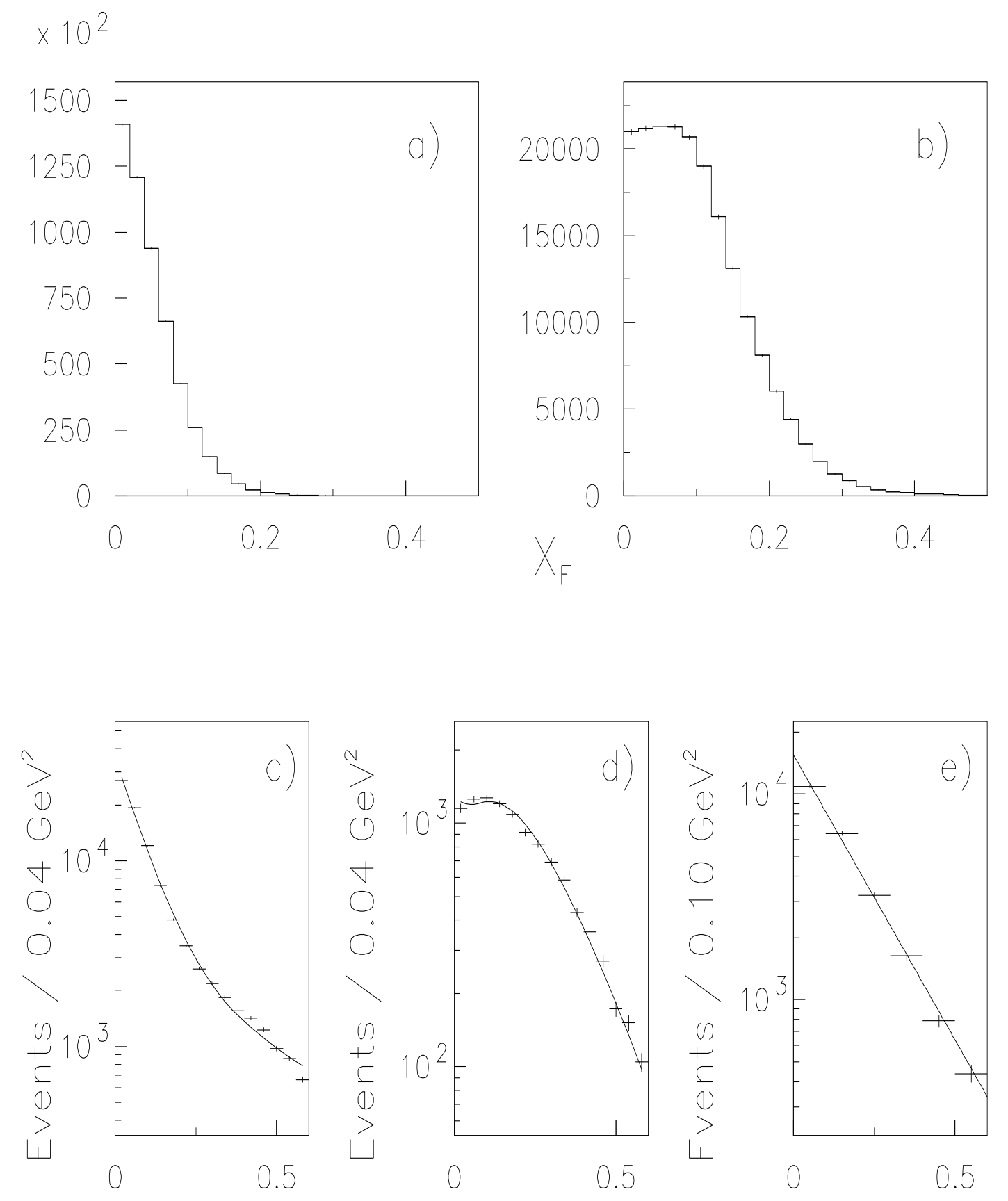

t GeV

Figure 2 

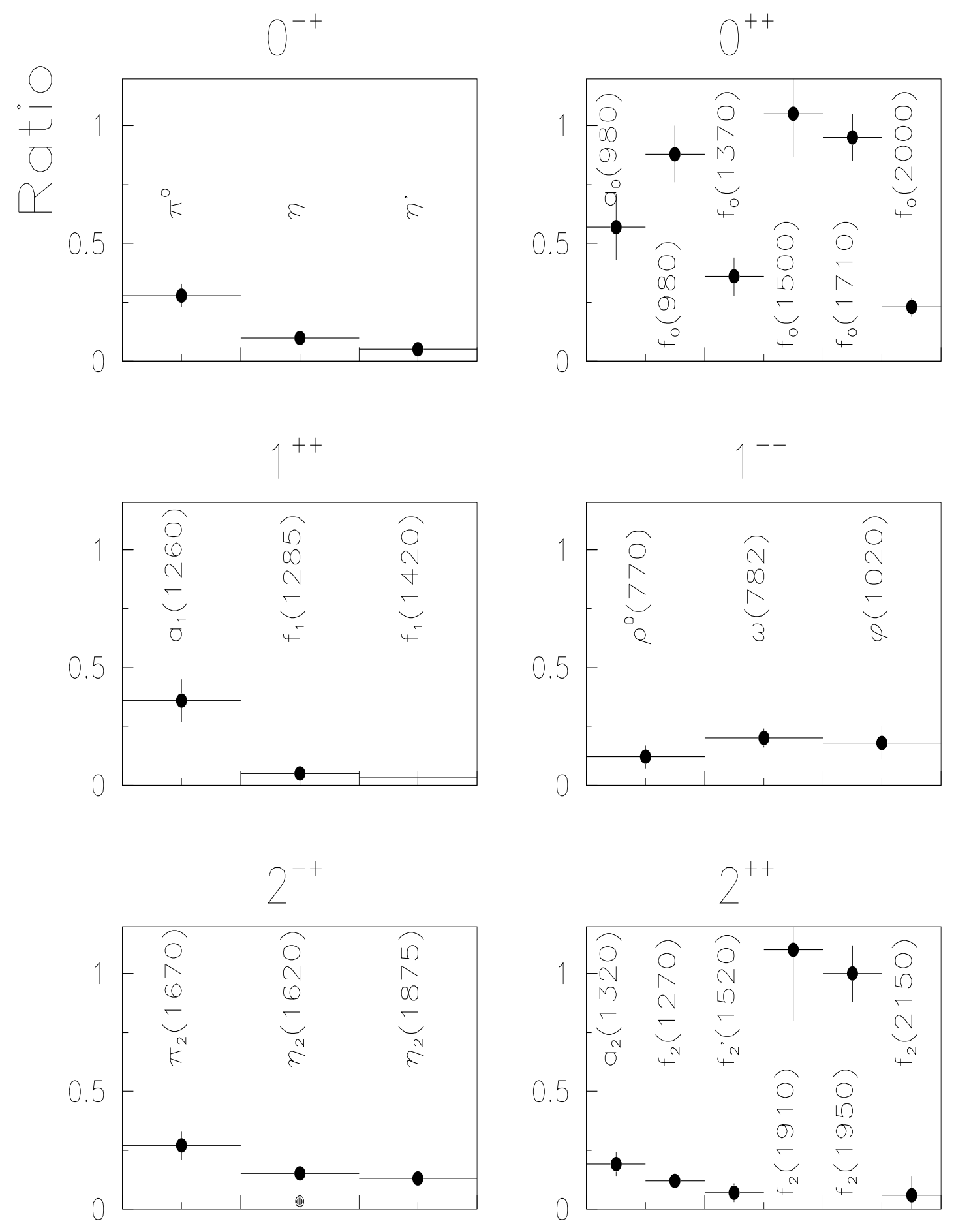

Figure 3 


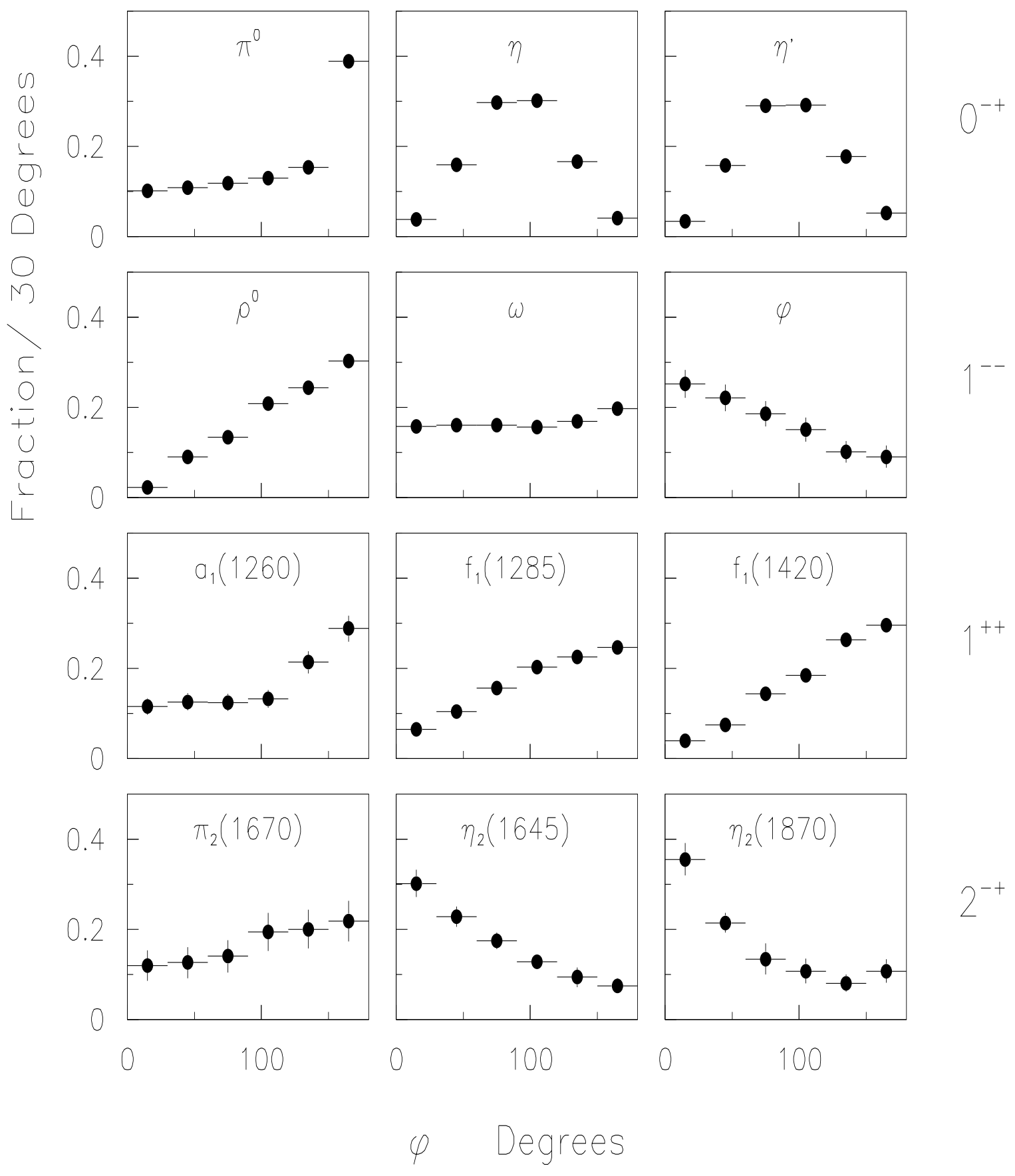

Figure 4 


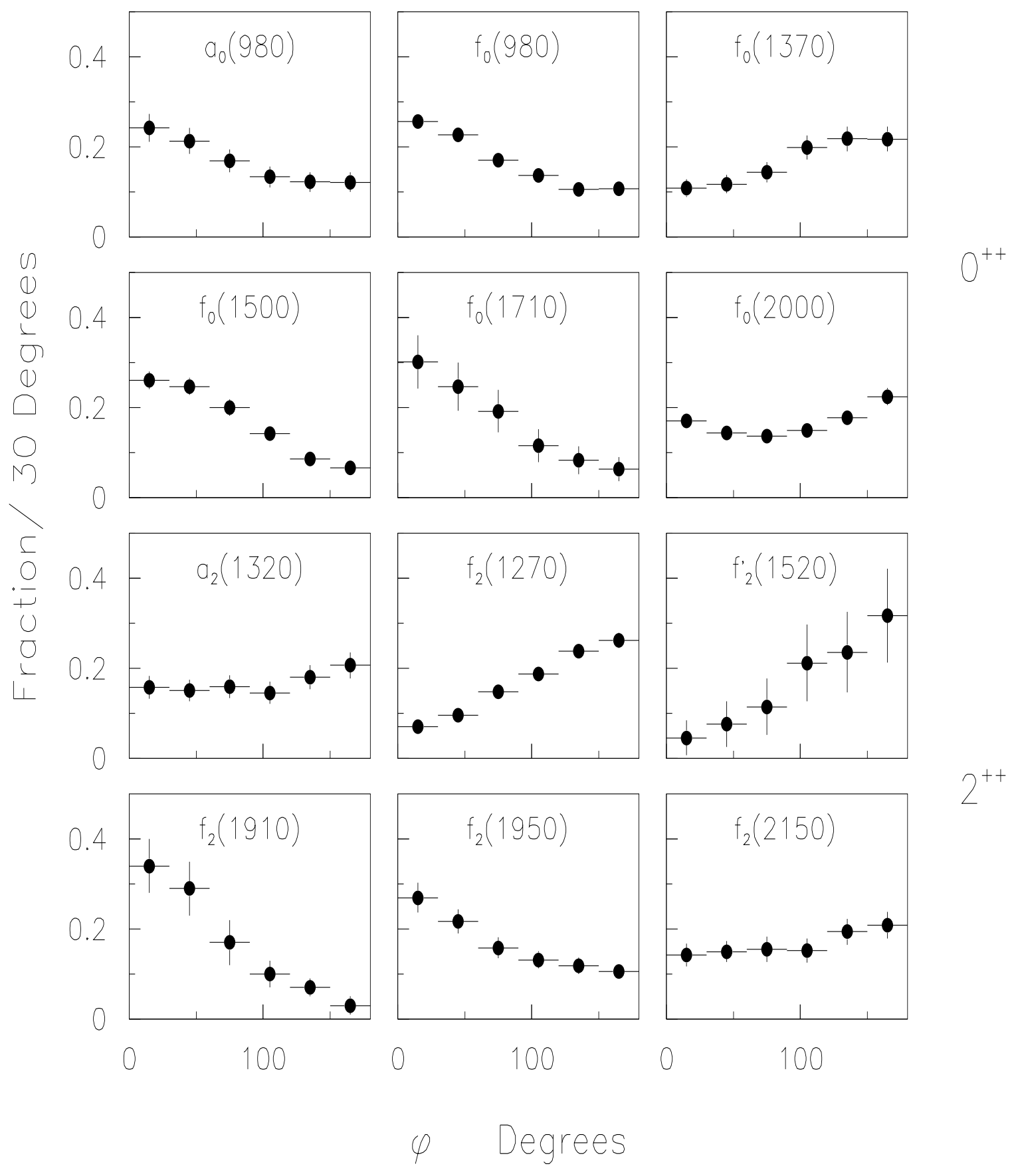

Figure 5 